\title{
MTRC correction algorithm for Bistatic ISAR in presence of constant Bistatic angle
}

\author{
Ning Han \\ Mechanical Technology Research Institute \\ Mechanical Engineering College \\ Shi Jiazhuang, China \\ haning1103@163.com
}

\author{
GeFang Wang \\ Mechanical Technology Research Institute \\ Mechanical Engineering College \\ Shi Jiazhuang, China
}

\author{
YanMei Lv \\ Mechanical Technology Research \\ Institute \\ Mechanical Engineering College \\ Shi Jiazhuang, China
}

\author{
MingFei Xia \\ Mechanical Technology Research \\ Institute \\ Mechanical Engineering College \\ Shi Jiazhuang, China
}

\author{
Dong Zhang \\ Mechanical Technology Research \\ Institute \\ Mechanical Engineering College \\ Shi Jiazhuang, China
}

\begin{abstract}
With the preliminary hypothesis of constant bistatic angle, matching filter pulse compression with non-coherent bistatic ISAR imaging mechanism is adopted and the reason of space target migration through resolution cell (MTRC) while bistatic inverse synthetic aperture radar (ISAR) imaging is researched. Then the correction algorithm based on Keystone transformation is put forward with the monostatic MTRC correction method as reference analysis thread. The validity of this algorithm is verified through space target ideal scatters simulation experiment.
\end{abstract}

Keywords-bistatic inverse synthetic aperture radar; moving through cell resolution; constant bistatic angle; direct sampling in medium frequency

\section{INTRODUCTION}

Over these years, with the rapid development of fourstrong-threaten, modern war puts more and more strict demand on the property of modern radar, so the monostatic radar faces more challenge and threat ${ }^{[1]}$. With the advantage in safety and anti-destroy, bistatic radar earns more and more attention ${ }^{[2]}$. Because bistatic inverse synthetic aperture radar (ISAR) imaging is not restricted by the moving format, bistatic ISAR imaging becomes an important research orientation with its inborn advantage of four-anti property ${ }^{[3-6]}$.

As the same problem in monostatic ISAR, MTRC also exists in bistatic ISAR. In order to solve this problem, the MTRC correction method in bistatic ISAR is researched ${ }^{[2]}$. But the method in [2] can only be used in bistatic turret model. As for the space target with more common character, the turret model can not be used any longer and the correction method becomes invalid.

The echo model of is constructed when target moving track and bistatic baseline is not in the same plane ${ }^{[7]}$. Based on the echo model, with the preliminary hypothesis of constant bistatic angle during imaging, the reason why MTRC exists is researched. Then the MTRC correction algorithm is put forward with the monostatic MTRC correction method as reference analysis thread. Finally, the algorithm is validated via simulation.

\section{ECHO MODEL WITH CONSTANT BISTATIC ANGLE}

The imaging geometry of moving target in bistatic ISAR is showed in Fig.1.

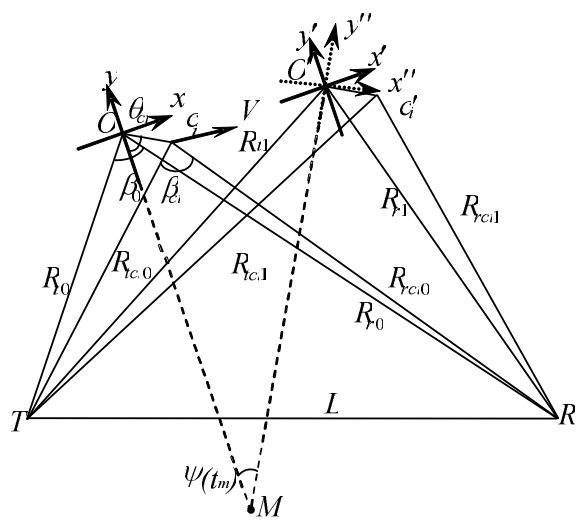

Figure 1. Imaging geometry of bistatic ISAR

In Fig.1, $\mathrm{T}$ is transmitting station; $\mathrm{R}$ is receiving station; axis $y$ is the bisector of target bistatic angle at the beginning of observation; $\beta_{0}$ is target bistatic angle, $\beta_{c i}$ is the bistatic angle of scatter $C_{i}$ in target, and approximately $\beta_{0} \approx \beta_{c i} ; O$ is the phase center; $\theta_{c i}$ is the angle of scatter $C_{i}$ position vector and axis y minus direction; $V$ is target moving velocity, $\mathrm{M}$ is the equivalent monostatic radar position at the beginning of observation; $\psi\left(t_{m}\right)$ is rotational angle of bisector during bistatic imaging. 
According to the model in Fig.1, [7] concludes that, whether the track of moving target and bistatic baseline belongs to the same plane or not, the changing distance of scatters can be expressed in (1) during the imaging process.

$$
\begin{aligned}
R_{i}\left(t_{m}\right) & =R_{r e f}\left(t_{m}\right)+R_{r a i}\left(t_{m}\right) \\
& =R_{r e f}\left(t_{m}\right)-2 r_{i} \cos \left[\theta_{i}+\psi\left(t_{m}\right)\right] \cos \left[\frac{\beta\left(t_{m}\right)}{2}\right]
\end{aligned}
$$

In (1), $t_{m}=m T(m=0,1,2, \mathrm{~L})$ is slow-time; $R_{r e f}\left(t_{m}\right)$ is the distance between phase center and transmitting-receiving bistatic stations which is the translational moving item; $R_{\text {roti }}\left(t_{m}\right)$ is the rotational distance item; $r_{i}$ is the distance between scatter $C_{i}$ and phase center $O ; \beta\left(t_{m}\right)$ is imaging bistatic angle.

From (1), it shows that, during bistatic ISAR imaging, the changing distance of scatter can also be divided into translational item $R_{\text {ref }}\left(t_{m}\right)$ and rotational item $R_{\text {roti }}\left(t_{m}\right)$. The only difference is that there is another half bistatic angle cosine in rotational item, which can only affect the concrete data of scatter rotational Doppler but can not change the essence that scatters are differentiated by different Doppler data. So, the principle of bistatic ISAR imaging is similar to monostatic ISAR imaging. The 2D image reconstruction can be accomplished by range compression, translational compensation, cross-range compression and so on ${ }^{[8]}$.

If some radar transmits LFM signal with the repetition circle $T$ and the bistatic radars are assumed to be ideal synchronized, the baseband echo of scatter $c_{i}$ after mediumfrequency direct sampling then desampling can be expressed as follows.

$$
S_{r i}\left(\hat{t}, t_{m}\right)=\sigma_{i} a_{d}\left(\hat{t}-\frac{R_{i}\left(t_{m}\right)}{c}, t_{m}\right) \times \exp \left[-j 2 \pi f_{0} \frac{R_{d}\left(t_{m}\right)}{c}\right]
$$

In (2), $\sigma_{c i}$ is the non-back scatter intensity of scatter $C_{i}$ in the ith rangebin; $a_{c i}$ is echocomplex envelope; $\hat{t}$ isfasttime; $t_{m}$ is slow-time; $f_{0}$ is radar carrier frequency.

\section{ANALYSIS OF BISTATIC MTRC GENERATION CAUSE}

For the bistatic radar system with fixed transmittingreceiving stations, during observation time, the bistatic angle of target can be approximated to be a constant without alteration. Based on this hypothesis, this paper analyzes the cause of bistatic MTRC and research according correction algorithm. For the convenience of narration in the latter of this paper, double passing distance of space target scatter $C_{i}$ should be changed to be as follows by (3)

$$
R_{c i}\left(t_{m}\right)=R_{t \mathrm{i}}\left(t_{m}\right)+R_{r i}\left(t_{m}\right)
$$

In (3), $R_{t i}\left(t_{m}\right)$ is the translational distance item and the effect of this item is assumed to be totally compensated by range alignment and initial phase correction; $R_{r i}\left(t_{m}\right)$ is rotational distance item; With the hypothesis that the bistatic angle is constant during imaging, $R_{r i}\left(t_{m}\right)$ can be expressed by (4) as follows.

$$
R_{r i}\left(t_{m}\right) \approx-2 r_{i} \cos \left[\theta_{c i}+\psi\left(t_{m}\right)\right] \cos \left[\beta_{0} / 2\right]
$$

Derivate (4), we can get the rotational velocity as (5)

$$
v_{r i}(t)=2 r_{i} \sin \left[\theta_{c i}+\psi\left(t_{m}\right)\right] \cos \left(\beta_{0} / 2\right) \psi^{\prime}\left(t_{m}\right)
$$

With (5), rotational distance $R_{r i}\left(t_{m}\right)$ can be approximated as (6)

$$
R_{r i}\left(t_{m}\right)=R_{r i 0}+v_{r i}\left(t_{m}\right) t_{m}
$$

In (6), $R_{r i 0}$ is the initial rotational distance.

If the rotational angle $\psi\left(t_{m}\right)$ of bisector can be approximated as (7)

$$
\psi\left(t_{m}\right)=\psi_{0}+\omega t_{m}
$$

In (7), $\psi_{0}$ is the initial rotational angle; $\omega$ is the rotational velocity of bisector. During the imaging process, the equation $\omega=\omega_{0}$ is thought to be founded。Now, substitute (7) into (5) and simplify it

$$
\begin{gathered}
v_{r i}\left(t_{m}\right)=2 x_{i} \cos \left(\psi_{0}+\omega_{0} t_{m}\right) \cos \left(\beta_{0} / 2\right) \omega_{0}- \\
2 y_{i} \sin \left(\psi_{0}+\omega_{0} t_{m}\right) \cos \left(\beta_{0} / 2\right) \omega_{0}
\end{gathered}
$$

For the above equation, in the time of $t_{m}=0$, spread $t_{m}$ via TAYLOR principle, and assume $\psi_{0}=0$, high-order item is neglected, we can get the follow equation

$$
\begin{aligned}
v_{r i}\left(t_{m}\right) & =2 x_{i} \cos \left(\beta_{0} / 2\right) \omega_{0}-2 y_{i} \cos \left(\beta_{0} / 2\right) \omega_{i}^{2} t_{m} \\
& =v_{r i 0}+a_{r i 0} t_{m} / 2
\end{aligned}
$$

In (9), $v_{r i 0}=2 x_{i} \cos \left(\beta_{0} / 2\right) \omega_{0}, a_{r i 0}=4 y_{i} \cos \left(\beta_{0} / 2\right) \omega_{f}^{2}$ 。

Substitute (9) into (6)

$$
R_{r i}\left(t_{m}\right)=R_{r i 0}+v_{r i 0} t_{m}+a_{r i 0} t_{m}^{2} / 2
$$

In general, the 3rd item in (10) is relatively smaller and can be neglected. Now, the rotational distance item of scatter $C_{i}$ during constant bistatic angle can be simplified to be as (11)

$$
R_{r i}\left(t_{m}\right)=R_{r i 0}+2 x_{i} \cos \left(\beta_{0} / 2\right) \omega_{0} t_{m}
$$

When the imaging bistatic angel is fixed to be $\beta_{0}$, the range and cross-range resolution of bistatic ISAR can be respectively expressed as (12)、(13) $)^{[3]}$

$$
\rho_{r}=\frac{c}{2 B \cos \left(\beta_{0} / 2\right)}
$$




$$
\rho_{a}=\frac{\lambda}{2 \Delta \theta \cos \left(\beta_{0} / 2\right)}
$$

In the above equations, $\rho_{r}$ is range resolution; $\rho_{a}$ is cross-range resolution; $C$ is the light speed in vacuum; $B$ is signal bandwidth; $\lambda$ is the transmitting signal wave; $\Delta \theta$ is rotational angle of bisector during imaging process.

In ISAR imaging, to get the higher cross-range resolution, rotational angel $\Delta \theta$ with some extent is needed. During the imaging process, if range migration $R_{r i}\left(t_{m}\right)$ of some scatter overpass range resolution $\rho_{r}$, then the scatter would generate MTRC. By analyzing (11) and $v_{r i 0}=2 x_{i} \cos \left(\beta_{0} / 2\right) \omega_{0}$, we can know that, in bistatic ISAR imaging, the rotational movement of bisector will generate the MTRC of scatter. And the larger the cross dimension of scatter is, the MTRC is more severe. Take $\lambda=2 \mathrm{~cm}, \mathrm{~B}=900 \mathrm{MHz}, x_{1}=5 \mathrm{~m}, x_{2}=4 \mathrm{~m}, \omega_{0}=0.1 \mathrm{rad} / \mathrm{s}$ as the example. Assume the bistatic angle is a constant as $40^{\circ}$, then the range resolution $\rho_{r}$ is $0.1774 \mathrm{~m}$, and if we expect the cross-range resolution to be $0.15 \mathrm{~m}$, the rotational angel has to be at least $4.0623^{\circ}$. Now, the range migration of scatter $x_{1}$ and scatter $x_{2}$ is respectively $0.6662 \mathrm{~m} 、 0.533 \mathrm{~m}$, which migrate 3.76 and 3 rangbins respectively. So, the range dimension of target image defocuses and the MTRC correction is needed to generate the clear image.

During the above analysis process, the 3rd item of (10) is neglected. The neglected item is related to range dimension. So to be strictly researched, the MTRC extent of scatter is not only related to the cross-range dimension but also related to the range dimension. But the square of imaging time is usually very small, so the neglecting of this item is totally acceptable.

\section{MTRC CORRECTION ALGORITHM}

As the effect of $R_{\mathrm{t}}\left(t_{m}\right)$ has been totally removed, we can change (2) from time zone to frequency zone and get (14) as follows

$$
S_{r}\left(f, t_{m}\right)=A(f) \sigma_{i} \exp \left[-j 2 \pi\left(f+f_{0}\right) \frac{R_{r i}\left(t_{m}\right)}{c}\right]
$$

In (14), $A(f)$ if the fourier transformation of $a_{c i}\left(\hat{t}-\frac{R_{r i}\left(t_{m}\right)}{c}, t_{m}\right)$. Multiply (14) with $A^{*}(f)$ to accomplish matching filter, we get (15) as follows

$$
S_{r}^{\prime}\left(f, t_{m}\right)=|A(f)|^{2} \sigma_{i} \exp \left[-j 2 \pi\left(f+f_{0}\right) \frac{R_{r i}\left(t_{m}\right)}{c}\right]
$$

Substitute (11) into the last equation

$$
\begin{aligned}
S_{r}^{\prime}\left(f, t_{m}\right) & =|A(f)|^{2} \&_{g} \exp \left[-j \frac{2 \pi}{c} f R_{r i 0}\right] \times \\
& \exp \left[\frac{-j 2 \pi}{c}\left(f+f_{0}\right) v_{r i 0} t_{m}\right]
\end{aligned}
$$

In (16), $8 / q=\sigma_{c i} \exp \left(-j 2 \pi R_{\text {rotio }} f_{0} / c\right)$.In (16), the $1 \mathrm{st}$ index item of spectrum is the position of target in time zero. The 2nd index item can be treated as different frequency with according different Doppler frequency shifts, which equals the MTRC of scatter.

In monostatic ISAR imaging, Keystone transformation is usually used to solve the MTRC problem. As the analysis thread to be refereed, this paper puts MTRC to be used in bistatic ISAR to correct MTRC. So, the virtual slow-time $\tau_{m}$ is defined and it satisfies (17)

$$
\left(f+f_{0}\right) t_{m}=f_{0} \tau_{m}
$$

Substitute (17) into (16), we can get the spectrum after Keystone transformation

$$
\begin{aligned}
S_{r}^{\prime}\left(f, \tau_{m}\right) & =|A(f)|^{2} \otimes_{c} \exp \left[-j \frac{2 \pi}{c} f R_{r i 0}\right] \times \\
& \exp \left[\frac{-j 2 \pi}{c} f_{0} v_{r i 0} \tau_{m}\right]
\end{aligned}
$$

From (18), we can conclude that, after Keystone transformation, the 2nd index item in echofast-time spectrum has nothing to do with frequency and the linear movement of echo envelope has been removed. Now, anti-transform (18) to be time-zone and we can get the echo after MTRC corrected.

In fact, Keystone transformation is the scale transformation of slow-time axis $t_{m}$. The transformation extent is related with fast-time $\hat{t}$. The initial data in (2) is aligned with rectangular shape, in $\hat{t}-t_{m}$ plane. After Keystone transformation, the data is changed to be in $\hat{t}-\tau_{m}$ plane and the shape is changed to be trapezium shape. To compress the data in azimuth dimension, the according algorithm is researched to change the data to be rectangular. To solve this problem, the scale-variant transformation, which can accomplish Keystone transformation, is studied. This method changes the data in $\hat{t}-t_{m}$ plane to data in frequency zone which is in accordance with virtual sloe-time $\tau_{m}$. Then, the data in frequency zone is inverse fourier transformed to $\hat{t}-\tau_{m}$ plane and the data is rectangularly aligned now. At he same time we get the rectangulaer-shape data, the interpolation operation is avoided, which efficiently improves algorithm counting efficiency. With the above descriped advantages, this paper adopts the method to finish the Keystone transformation of data. In general, the MTRC correction method for bistatic ISAR of space target could be divided into such steps:

Step1: For the complex baseband echo, the match-filter pulse compression process is firstly executed to get the initial data without MTRC correction;

Step2: The translational compensation is carried out to remove the effect of translational item $R_{t i}\left(t_{m}\right)$;

Step3 : The scale-variant fourier transformation and inverse fourier transformation is accomplished to finish the 
Keystone transformation of echo data and get the data after MTRC correction;

Step4: Azimuth compression of data after correction is finished to get 2D image of the target.

The above analysis and study is only aimed at sole scatter MTRC correction method. In practical imaging process, target is composed of many scatters and the real echo could be treated as linear composition of the echo of every scatter. So, the algorithm researched in this paper could easily extend to the real target imaging process.

\section{SIMULATION EXPERIMENT}

This paper makes research on space target imaging. So, the simulation experiment is also aimed at space target bisatic ISAR imaging. The simulation scene and scatter model is showed in Fig. 1 and Fig.2 respectively.

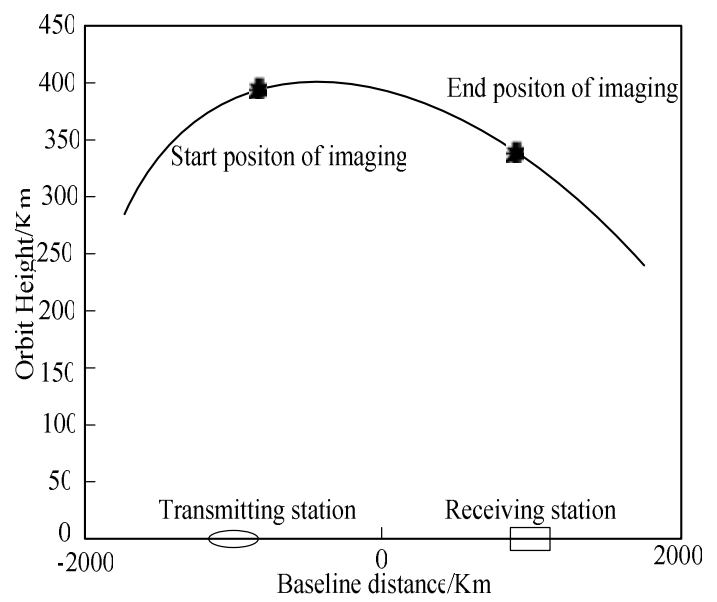

Figure 1. simulation scene

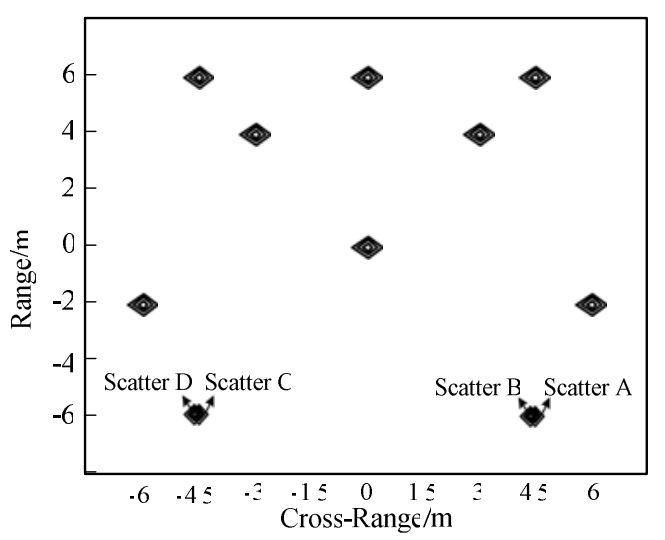

Figure 2. Scatter model

In Fig.1, circle stands for the transmitting station and rectangle stands for receiving station. The height of target circle is about $300 \mathrm{Km}$, and the double passing distance is $4 \mathrm{Km} / \mathrm{s}$. Target circle is generated by STK software, and the circle parameters is set to guarantee the bistatic angle keeps constant during imaging process. In simulation, target is substituted by scatter model. As in Fig.2, the RCS of target is set to be $6 \mathrm{~m} 2$. Because the scatters energy in wideband imaging is separated, so the equivalent RCS of every scatter is $0.5 \mathrm{~m} 2$. With the imaging resolution cell taken into consideration, the RCS level is normal in space target imaging. The signal-to-noise ratio of this imaging circle could be easily counted.

\section{TABLE 1 PARAMETERS OF BISTATIC RADAR IN SIMULATION}

\begin{tabular}{cccc}
\hline parameter & index & parameter & Index \\
\hline Carrier frequency & $8 \mathrm{GHz}$ & $\begin{array}{c}\text { Pulse width } \\
\text { Sampling } \\
\text { frequency } \\
\text { Signal bandwidth }\end{array}$ & $900 \mathrm{MHz}$ \\
$\begin{array}{c}\text { Bulse repetine } \\
\text { frequency }\end{array}$ & $150 \mathrm{~Hz}$ & $\begin{array}{c}\text { length } \\
\text { frion }\end{array}$ & $2000 \mathrm{Km}$ \\
\hline
\end{tabular}

Fig.1 shows the radar parameters of bistatic radar in simulation. During imaging simulation, the rotational velocity $\omega_{0}$ of bisector is assumed to be $0.1 \mathrm{rad} / \mathrm{s}$ and the bistatic angle is $120^{\circ}$. Now, according to (12), the image resolution is $0.33 \mathrm{~m}$. The cross-distance between A 、 B and C、 D is $0.15 \mathrm{~m}$ respectively. To differentiate the four scatters in cross-distance, the needed imaging rotational angle is $0.25 \mathrm{rad}$ counted by (13) according to the parameters in table 1 . Within these imaging conditions, scatter A moves $1.125 \mathrm{~m}$ during imaging process and migrates about 3.4 range cells because the rotation of bisector. So the MTRC correction is needed to get clear 2D image. Fig.3 and Fig. 4 demonstrate the bistatic 2D images before and after MTRC.

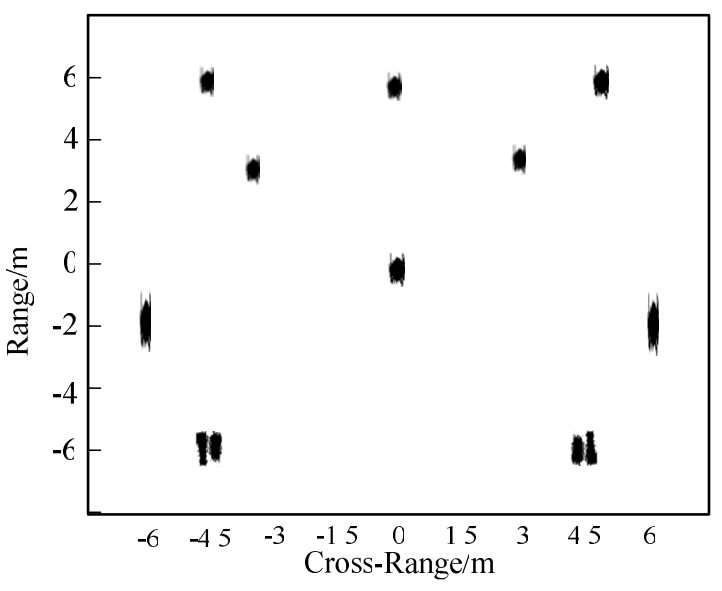

Figure 3. 2D image before MTRC correction

As in Fig.3, because the rang resolution is $0.33 \mathrm{~m}$, the $2 \mathrm{D}$ image can totally differentiate the 5 scatters which are in different range cells. And because the rotational angle during imaging process is $0.25 \mathrm{rad}$, scatters A、B and scatters C、D could be differentiated in Doppler dimension. Fig.3 still shows that target scatters moves through some range resolution cells that is MTRC happened. And the larger the cross-range of scatter is, the MTRC is more severe, which is in accordance with the previous theoretical analysis.

Now, the 2D image is corrected via the algorithm researched in section 3 and we get the image after correction in Fig.4 


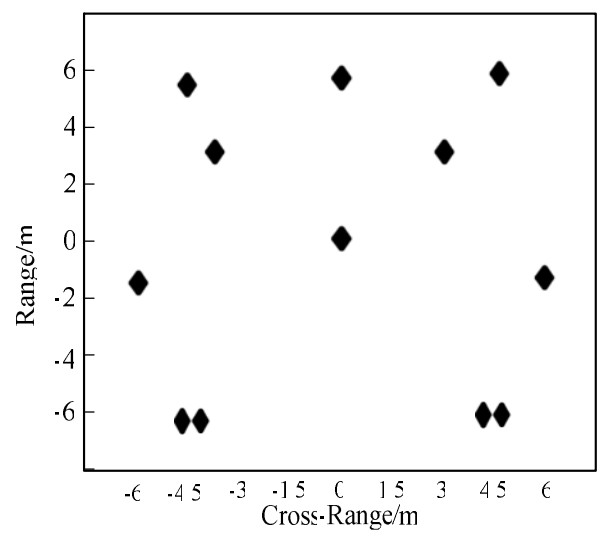

Figure 4. 2D image after MTRC correction

As shown in Fig.4, MTRC of scatters has been totally corrected and we get the clear image, which verifies the algorithm.

\section{CONCLUSION}

With the assumption that the bistatic angle keeps constant during imaging process, the character of complex bistatic ISAR baseband echo is researched and the conclusion that the rotation of bisector during imaging process could lead to the MTRC is got. Keystone transformation is adopted to remove the MTRC in target range profile. Finally, space target model with ideal scatters is adopted in simulation experiment. The experiment results show that Keystone transformation could effectively remove MTRC in bistatic ISAR.

\section{REFERENCES}

[1]. Sword C.K., Simaan M., Kamen E.W. Multiple target angle tracking using sensor array outputs[J]. IEEE Transactions on Aerospace and Electronic Systems, 1990, 26(2):367-373.

[2]. Marco Martorella, James Palmer, John Homer, et al. On Bistatic Inverse Synthetic Aperture Radar[J]. IEEE Transactions on Aerospace and Electronic Systems, 2007(3):1125-1134.

[3]. WANG Ge-fang, CHEN Guo-shun, WANG Xue-ming, et al. Design of Equipment Parts Based on Object Reverse Engineering Technologies[J]. Ordnance Industry Automation, 2009, 28(4):12-14.

[4]. Sword C.K., Simaan M., Kamen E.W. Multiple Target Angle Tracking Using Sensor Array Outputs[J]. IEEE Transactions on Aerospace and Electronic Systems, 1990, 26(2):367-373.

[5]. Blyakhman A.B. , Runova I.A. Forward Scattering Radiolocation Bistatic Rcs and Target Detection[C]. The Record of the 1999 IEEE Radar Conference, 1999:203-208.

[6]. Dong Jian, Shang Chao-xuan, Gao Mei-guo, et al. Research on bistatic ISAR speed compensation of space target[J]. Journal of China Academy of Electronics and Information Technology, 2010, (1):78-85..

[7]. Zhu Ren-fei, Luo Ying, Zhang Qun, et al. Analysis of bistatic inverse synthetic aperture radar imaging[J]. Modern Radar, 2011, 33(9):33-38..

[8]. Sugiyama M, Imaizumi D, Ogawa H. Subspace Information Criterion for Image Restoration-Optimizing Parameters in Linear Filters[J]. IEICE Trans on Information and Systems, 2001, 84(9):1249-1256. 